\title{
Assessment of motion error for frame-based and noninvasive mask-based fixation using the Leksell Gamma Knife Icon radiosurgery system
}

\author{
Arthur Carminucci, MD, ${ }^{1}$ Ke Nie, $\mathrm{PhD},{ }^{2}$ Joseph Weiner, $\mathrm{MD},{ }^{2}$ Eric Hargreaves, $\mathrm{PhD},{ }^{1}$ and \\ Shabbar F. Danish, MD' \\ ${ }^{1}$ Department of Neurological Surgery, Rutgers University; and 2Department of Radiation Oncology, Rutgers Cancer Institute of \\ New Jersey, Rutgers Robert Wood Johnson Medical School, New Brunswick, New Jersey
}

OBJECTIVE The Leksell Gamma Knife Icon (GK Icon) radiosurgery system can utilize cone-beam computed tomography (CBCT) to evaluate motion error. This study compares the accuracy of frame-based and frameless mask-based fixation using the Icon system.

METHODS A retrospective cohort study was conducted to evaluate patients who had undergone radiosurgery with the GK Icon system between June and December 2017. Patients were immobilized in either a stereotactic head frame or a noninvasive thermoplastic mask with stereotactic infrared (IR) camera monitoring. Setup error was defined as displacement of the skull in the stereotactic space upon setup as noted on pretreatment CBCT compared to its position in the stereotactic space defined by planning MRI for frame patients and defined as skull displacement on planning CBCT compared to its position on pretreatment $\mathrm{CBCT}$ for mask patients. For frame patients, the intrafractionation motion was measured by comparing pretreatment and posttreatment $\mathrm{CBCT}$. For mask patients, the intrafractionation motion was evaluated by comparing pretreatment $\mathrm{CBCT}$ and additional $\mathrm{CBCT}$ obtained during the treatment. The translational and rotational errors were recorded.

RESULTS Data were collected from 77 patients undergoing SRS with the GK Icon. Sixty-four patients underwent frame fixation, with pre- and posttreatment $\mathrm{CBCT}$ studies obtained. Thirteen patients were treated using mask fixation to deliver a total of 33 treatment fractions. Mean setup and intrafraction translational and rotation errors were small for both fixation systems, within $1 \mathrm{~mm}$ and $1^{\circ}$ in all axes. Yet mask fixation demonstrated significantly larger intrafraction errors than frame fixation. Also, there was greater variability in both setup and intrafraction errors for mask fixation than for frame fixation in all translational and rotational directions. Whether the GK treatment was for metastasis or nonmetastasis did not influence motion uncertainties between the two fixation types. Additionally, monitoring IR-based intrafraction motion for mask fixation-i.e., the number of treatment stoppages due to reaching the IR displacement threshold-correlated with increasing treatment time.

CONCLUSIONS Compared to frame-based fixation, mask-based fixation demonstrated larger motion variations. The variability in motion error associated with mask fixation must be taken into account when planning for small lesions or lesions near critical structures.

https://thejns.org/doi/abs/10.3171/2018.7.GKS181516

KEYWORDS Gamma Knife; motion error; GK Icon; frame fixation; mask fixation; stereotactic radiosurgery

$\mathrm{T}$ HE high dose of radiation delivered via stereotactic radiosurgery (SRS) requires accurate and reproducible immobilization of the head.? Accuracy within a range of $1-2 \mathrm{~mm}$ is essential to limit irradiation of surrounding anatomical structures. A rigid stereotactic head frame has traditionally been used for fixation in Gamma Knife (GK) radiosurgery. ${ }^{20}$ Frame-based fixation allows for precise delivery of a single high-dose fraction of radiation treatment. Motion error associated with frame-based fixation is estimated to be $\leq 1-1.5 \mathrm{~mm} .{ }^{12}$

New SRS strategies for the treatment of large intracranial lesions have trended toward the use of multifractionation stereotactic radiotherapy (SRT) treatments. But head frame placement is invasive, involving 4-point fixation us-

ABBREVIATIONS CBCT = cone-beam computed tomography; GK = Gamma Knife; IR = infrared; LINAC = linear accelerator; SRS = stereotactic radiosurgery; SRT = stereotactic radiotherapy.

SUBMITTED May 30, 2018. ACCEPTED July 24, 2018.

INCLUDE WHEN CITING DOI: 10.3171/2018.7.GKS181516. 
ing screws secured into the patient's skull, and thus fractionated treatments with head frames are difficult. Frameless fixation is better suited for fractionated treatments. Multiple mask-based modalities have been tested for fractionated treatments using linear accelerator (LINAC)based systems..$^{3-6,16}$ More recently, noninvasive fixation has been attempted on modified versions of the Leksell GK system..$^{9,15,17}$ The latest version, Leksell Gamma Knife Icon (GK Icon), is capable of frameless, noninvasive fixation via a thermoplastic mask. The system utilizes conebeam computed tomography (CBCT) and high-definition infrared (IR)-based motion monitoring to allow for frameless fixation. Thus, the GK Icon can be utilized for fractionated SRT-based GK treatment.

As fractionated GK radiosurgery becomes more prevalent, a better understanding of how motion error associated with mask fixation compares to the uncertainties of frame fixation becomes necessary. In this study, we utilized CBCT to evaluate motion error for frame-based and frameless mask-based fixation using the GK Icon. Motion uncertainties were measured in the translational and rotational directions.

\section{Methods \\ Patients}

The study was conducted with the approval of our institutional review board and represents a retrospective cohort study. Between June and December 2017, 77 patients underwent GK radiosurgery using the GK Icon system. Patients were immobilized in either the standard Leksell head frame or the thermoplastic mask for treatment. The decision to treat patients with either a head frame or the thermoplastic mask was based on the lesion's characteristics, treatment plan, and discretion of the treating neurosurgeon and radiation oncologist.

\section{Clinical Workflow for GK Icon Treatment}

For patients undergoing GK treatment with rigid fixation, the Leksell Model G head frame (Elekta AB) was used. Head frames were placed with 4 pins secured into the patient skull by a neurosurgeon prior to the start of the procedure. Same-day MRI studies with the indicator box on were acquired for treatment planning purposes. Prior to treatment, a pretreatment CBCT scan was acquired to obtain the patient position. Following completion of the treatment, a posttreatment CBCT study was obtained. Setup error in the frame-based fixation group was defined as displacement of the skull in the stereotactic space upon setup as noted on pretreatment CBCT compared to its position in the stereotactic space defined by planning MRI. Intrafractionation motion was assessed by comparing the pre- and posttreatment CBCT.

At our institution, patients undergoing immobilization with a thermoplastic mask were brought into the GK center a day before the start of their treatment. At this time, the thermoplastic mask was molded to the contours of the patient's face. A planning CBCT was acquired to obtain the stereotactic reference space. On the day of treatment, the mask was reapplied and a pretreatment CBCT was captured. A single reflective optical marker was placed on the patient's nose tip as a stable anatomical reference point. The pretreatment CBCT was registered to the planning CBCT. Registration information was then transferred to the treatment machine, which allows the patient position to be matched to the stereotactic reference space. Once treatment was initiated, patient position was monitored by IR camera-based tracking at the nose tip. If the motion detected was beyond a preset threshold (default of $1.5 \mathrm{~mm})$, the treatment would be halted. Another set of treatment CBCTs would be needed and registered to the planning CBCT prior to reinitiating treatment. Setup error was assessed by comparing the planning CBCT to the pretreatment $\mathrm{CBCT}$, whereas the intrafractionation motion was compared between treatment CBCT(s) and pretreatment CBCT. Additionally, we assessed IR displacement as the number of treatment stoppages due to reaching the displacement threshold compared to the treatment time and to the treatment fraction number.

\section{Statistical Analysis}

All images were transferred to commercial software (Velocity, Varian) for processing. Rigid transformation was used to align the patient skull from one image set to another. Translational and rotational displacements were quantified by setting the center of the frame $(100,100,100$ at the GK stereotactic coordinates) as the center of rotation and the origin of the coordinate system. The absolute translational errors for each immobilization system were reported in the $\mathrm{x}$ - (left to right), $\mathrm{y}$ - (cranial-caudal), and $\mathrm{z}$ (anterior-posterior) axes, respectively. Absolute rotational errors were measured in the $\mathrm{x}$ - (pitch), $\mathrm{y}$ - (yaw), and z- (roll) axes. Error was reported as the mean \pm standard deviation. The group mean comparison between frame-based and mask-based fixation was performed using a nonparametric Mann-Whitney U-test. The variation between two groups was compared using Levene's test. Since most of our cases were brain metastases, which can include small lesion sizes and in which the coverage may be more vulnerable to motion errors, we also performed a comparison between metastases and nonmetastases. The comparison of error was performed using a nonparametric KruskalWallis test with Dunn's multiple comparisons. A p value less than 0.05 was considered statistically significant. For the mask cases, the assessment of IR displacement as the number of treatment stoppages due to reaching the displacement threshold compared to treatment time and to treatment fraction number was also reported.

\section{Results}

\section{Patient Demographics}

The CBCT scans from a total of 77 patients were analyzed. Patient characteristics are summarized in Table 1. Sixty-four patients underwent frame fixation, with preand posttreatment CBCTs obtained. Thirteen patients were treated with mask fixation. The total number of treatment fractions for the mask group was 33. Five patients were treated with a single fraction, 2 with 2 fractions, 3 with 3 fractions, and 3 were treated over the course of 5 fractions. Patients were treated for a variety of intracranial lesions. The lesion most commonly treated was cerebral 
TABLE 1. Summary of characteristics in 77 patients who underwent GK treatment

\begin{tabular}{lc}
\hline \multicolumn{1}{c}{ Parameter } & No. \\
\hline Type of lesion & \\
\hline Cerebral metastasis & 53 \\
\hline Meningioma & 10 \\
\hline Trigeminal neuralgia & 4 \\
\hline Schwannoma & 4 \\
\hline Pituitary adenoma & 3 \\
\hline AVM & 2 \\
\hline Glomus tumor & 1 \\
\hline Type of fixation & 64 \\
\hline Frame & 13 \\
\hline Mask & $66 \pm 50$ \\
\hline Average treatment time per fraction (mins) & $55 \pm 25$ \\
\hline Frame
\end{tabular}

$\mathrm{AVM}=$ arteriovenous malformation.

metastasis (68.8\%). Average treatment times for frame and mask fixation for each fraction were $66 \pm 50$ and $55 \pm 25$ minutes, respectively. There was no statistical difference in treatment times between the two fixation groups.

\section{Setup Error}

Translational and rotational setup errors for framebased and mask-based fixation are summarized in Table 2. The mean absolute translational error for both fixation groups was small, $1 \mathrm{~mm}$ or less. There was no statistical difference in the average translational error between frame- and mask-based fixations. The average rotational error for both groups was also small, less than $1^{\circ}$. Average rotational error in the z-axis was statistically different between frame- and mask-based fixation $\left(0.18^{\circ} \pm 1.3^{\circ}\right.$ vs $\left.0.98^{\circ} \pm 1.24^{\circ}, \mathrm{p}<0.002\right)$. There was no difference in the rotational $\mathrm{x}$ - and $\mathrm{y}$-axis error. Scatter plots of translational and rotational setup errors for frame- and mask-based fixation are shown in Fig. 1. The maximal displacement of translational error was higher for mask fixation across all three axes (Fig. 1A). Similarly, the maximal displacement of rotational error was higher for mask fixation across all three axes (Fig. 1B). Analysis of the variance in error using Levene's test demonstrated significantly greater variance in the mask fixation than in frame fixation in all translational and rotational directions (Supplemental Table 1).

\section{Intrafraction Error}

Translational and rotational absolute intrafraction errors for frame-based and mask-based fixation are summarized in Table 3. Both translational and rotational errors in all directions for frame fixation were minimal. However, the mean absolute error for mask fixation was higher than that for frame fixation in all three directions for both translational and rotational errors. Additionally, the variations of translational error (Fig. 2A) and rotational error (Fig. 2B) were much greater for mask fixation across all
TABLE 2. Translational and rotational absolute setup error

\begin{tabular}{ccc}
\hline & \multicolumn{2}{c}{ Mean \pm SD } \\
\cline { 2 - 3 } Setup Error & \multicolumn{1}{c}{ Frame } & Mask \\
\hline Translational $(\mathrm{mm})$ & & $0.55 \pm 0.78$ \\
\hline x-axis & $0.25 \pm 0.25$ & $0.52 \pm 0.65$ \\
\hline$y$-axis & $0.20 \pm 0.17$ & $1.00 \pm 1.46$ \\
\hline z-axis & $0.33 \pm 0.24$ & \\
\hline Rotation $\left(^{\circ}\right)$ & & $0.49 \pm 0.58$ \\
\hline$x$-axis & $0.36 \pm 0.30$ & $0.56 \pm 0.89$ \\
\hline$y$-axis & $0.24 \pm 0.21$ & $0.98 \pm 1.24^{*}$ \\
\hline z-axis & $0.18 \pm 0.13^{*}$ & \\
\hline$S D=$ standard deviation. & & \\
${ }^{*} p<0.05$. & &
\end{tabular}

three axes. Levene's test demonstrated significantly greater variance in the mask fixation than in the frame fixation in all translational and rotational directions (Supplemental Table 1).

\section{Metastases Versus Nonmetastases}

A subanalysis of setup and intrafraction errors was performed by comparing patients with metastases to those with nonmetastases using a one-way ANOVA analysis of absolute error. There was no statistical difference in the setup translational error for metastases compared to nonmetastases for either frame or mask fixation. There was also no significant difference in $\mathrm{x}$ - and $\mathrm{y}$-rotational errors between the fixation groups. The z-rotational error only differed between frame metastasis and mask metastasis on multiple comparisons analysis (Supplemental Fig. 1). Mask fixation demonstrated significantly higher intrafraction error than frame fixation for both metastases and nonmetastases patients in both the translational and rotational directions. There was no significant difference between frame fixation for metastasis and frame fixation for nonmetastasis. Similarly, there was no significant difference between mask fixation for metastasis and mask fixation for nonmetastasis in any translational or rotational direction (Supplemental Fig. 2).

\section{Infrared-Based Intrafraction Motion}

For patients undergoing GK treatment with mask-based fixation, we also measured IR-based intrafraction motion. Infrared tracking was used to monitor patient head movement during the course of radiation delivery. When IR displacement at the nose tip reached a threshold of $1.5 \mathrm{~mm}$, treatment was stopped and a CBCT scan was obtained to reassess for motion error. The number of treatment stoppages due to reaching the IR displacement threshold correlated with increasing treatment time (Fig. 3A). These results trended toward but did not reach statistical significance. The number of treatment stoppages based on the treatment fraction number was also measured (Fig. 3B). On average, the number of times reaching the displacement threshold decreased between fractions 1 to 3 . This was followed by an upward trend once 4 or more fractions were delivered. 


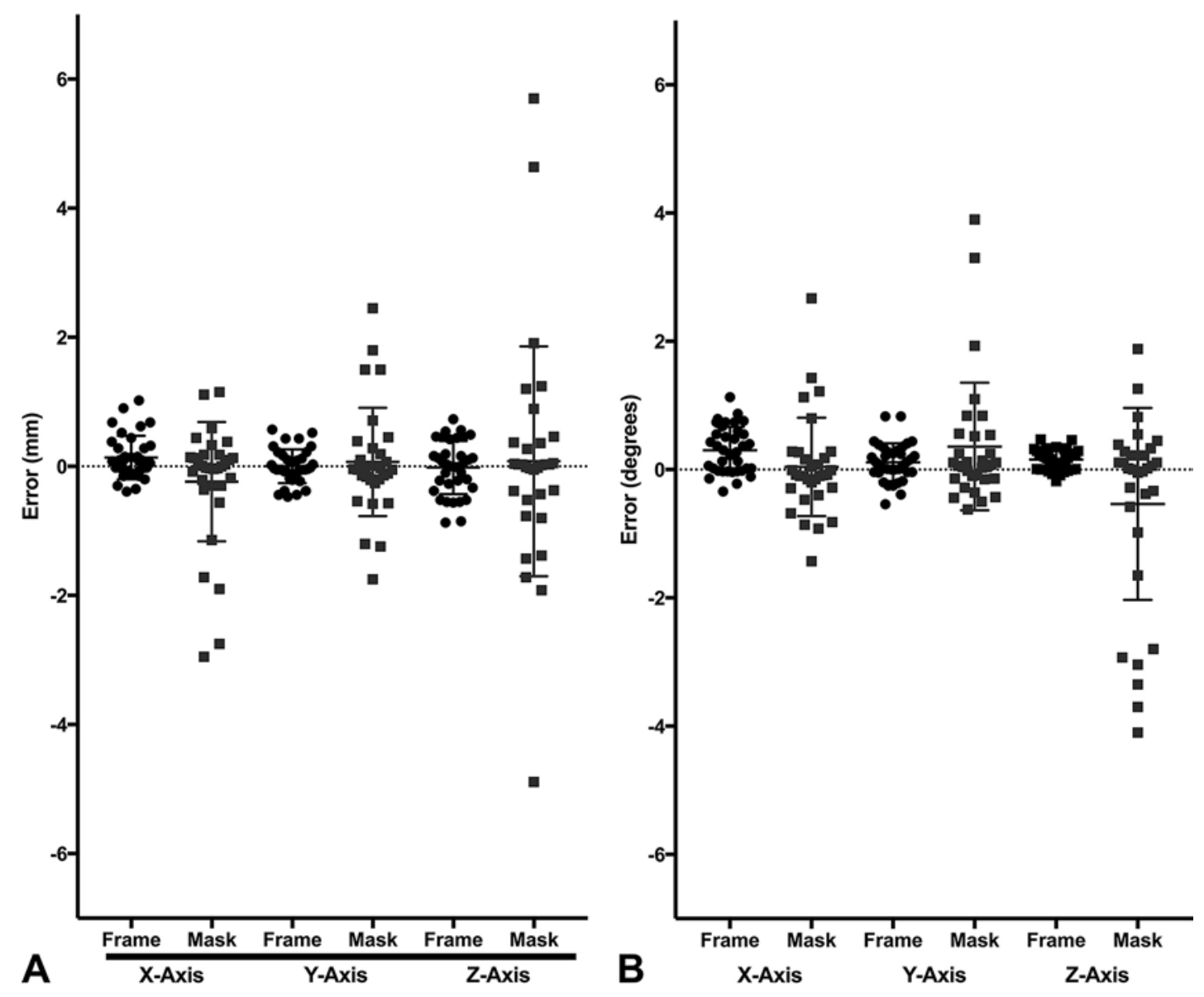

FIG. 1. A: Scatter plot of translational setup errors for frame- and mask-based fixation. Maximal translation displacement for frame-based fixation was $1.02,0.57$, and $-0.87 \mathrm{~mm}$ in the $x-, y-$, and $z$-axis, respectively. Maximal translation displacement for mask-based fixation was $-2.95,2.45$, and $5.7 \mathrm{~mm}$ in the $x-, y-$-, and z-axis, respectively. B: Scatter plot of rotational setup errors for frame- and mask-based fixation. Maximal rotational displacement for frame-based fixation was $1.13^{\circ}, 0.83^{\circ}$, and $0.47^{\circ}$ in the $x-$, $y-$, and z-axis, respectively. Maximal rotational displacement for mask-based fixation was $2.67^{\circ}, 3.9^{\circ}$, and $-4.1^{\circ}$ in the $x-, y-$, and z-axis, respectively.

\section{Discussion}

Stereotactic radiosurgery requires accurate and precise delivery of high-dose radiation to a specified target while minimizing potentially toxic radiation to the surrounding tissue. Rigid fixation with a stereotactic head frame al-

TABLE 3. Translational and rotational absolute intrafraction error

\begin{tabular}{ccc}
\hline & \multicolumn{2}{c}{ Mean \pm SD } \\
\cline { 2 - 3 } Intrafraction Error & Frame & Mask \\
\hline Translational $(\mathrm{mm})$ & & \\
\hline$x$-axis & $0.05 \pm 0.04^{*}$ & $0.75 \pm 0.73^{*}$ \\
\hline$y$-axis & $0.03 \pm 0.02^{*}$ & $0.73 \pm 0.60^{*}$ \\
\hline z-axis & $0.08 \pm 0.07^{*}$ & $1.67 \pm 2.12^{*}$ \\
\hline Rotational $\left({ }^{\circ}\right)$ & & \\
\hline$x$-axis & $0.03 \pm 0.03^{*}$ & $0.73 \pm 0.62^{*}$ \\
\hline$y$-axis & $0.07 \pm 0.07^{*}$ & $0.76 \pm 0.63^{*}$ \\
\hline$z$-axis & $0.07 \pm 0.13^{*}$ & $1.44 \pm 1.79^{*}$ \\
\hline
\end{tabular}

${ }^{*} p<0.05$ lows for immobilization of the patient's head and serves as a stereotactic coordinate system for target localization. ${ }^{7}$ Typically, submillimeter accuracy is achievable for singlefraction treatment of small lesions. ${ }^{11}$ Limitations of frame fixation include the invasiveness of frame placement and the difficulty in performing multifraction treatments. The difficulty in performing fractioned treatments with frame fixation is attributable to the impracticality of either leaving a patient in the frame for several days or having to reapply the frame several times over the course of treatment. Alternatively, frameless fixation through the use of a thermoplastic mask is noninvasive and makes multifraction treatments more feasible. The GK Icon is capable of frameless fixation through the use of CBCT. For frameless cases, CBCT provides a stereotactic coordinate system for target localization through coregistration of the planning MRI and reference CBCT. Additionally, CBCT can be used to verify patient position during setup prior to irradiation. ${ }^{1}$ In this study, we utilized CBCT to compare motion uncertainties between frame-based fixation and mask-based fixation while using the GK Icon.

Setup error based on CBCT measurements was mea- 


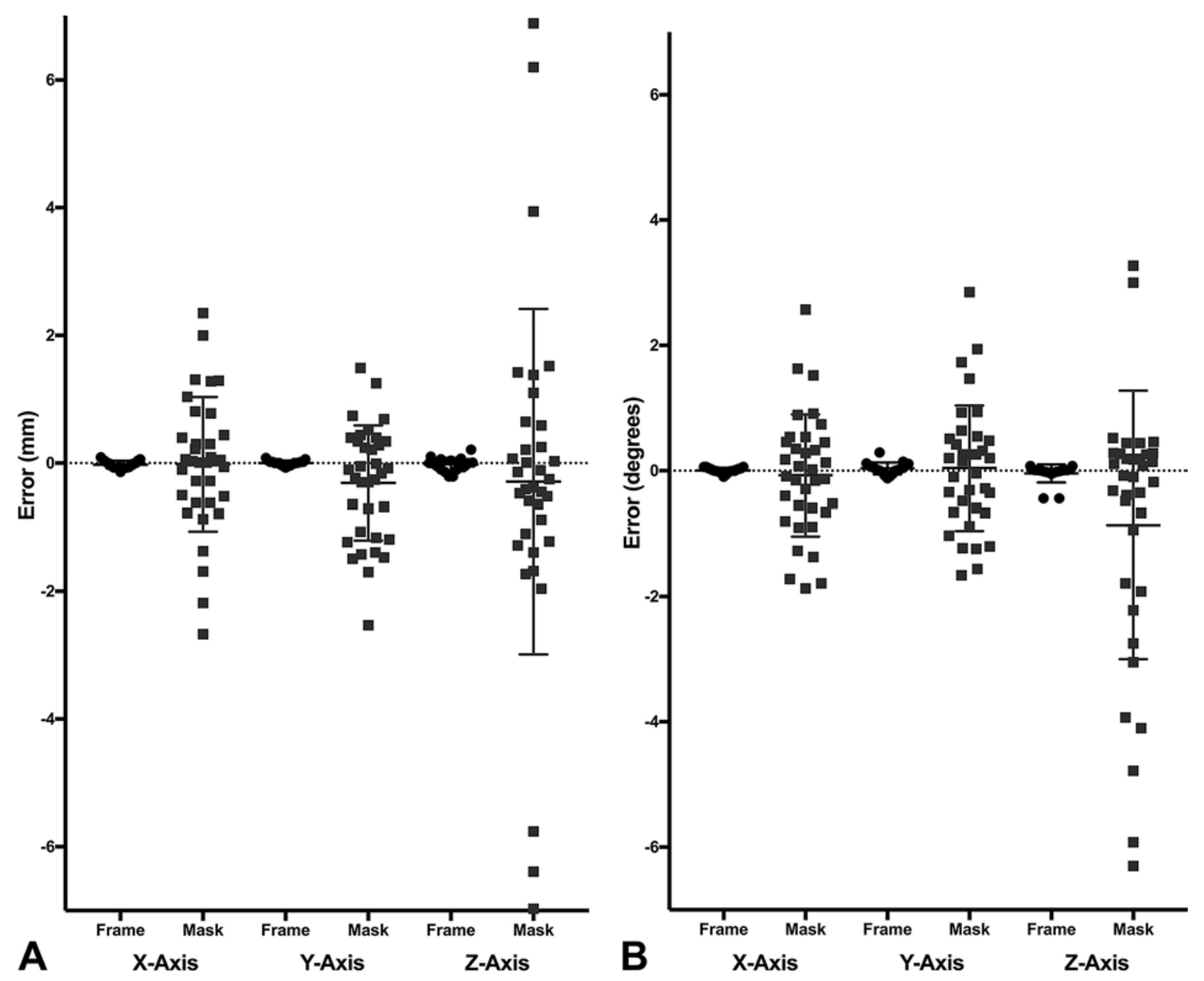

FIG. 2. A: Scatter plot of intrafraction setup errors for frame- and mask-based fixation. Maximal translational displacement for frame-based fixation was $-0.14,0.08$, and $0.21 \mathrm{~mm}$ in the $x-, y-$, and $z$-axis, respectively. Maximal translational displacement for mask-based fixation was $-2.67,-2.53$, and $-6.97 \mathrm{~mm}$ in the $x-, y-$, and $z$-axis, respectively. B: Scatter plot of rotational intrafraction errors for frame- and mask-based fixation. Maximal rotational displacement for frame-based fixation was $-0.09^{\circ}, 0.29^{\circ}$, and $-0.44^{\circ}$ in the $x-, y-$, and $z$-axis, respectively. Maximal rotational displacement for mask-based fixation was $2.57^{\circ}, 2.85^{\circ}$, and $3.27^{\circ}$ in the $x-, y-$, and $z$-axis, respectively.

sured for patients undergoing frame-based and maskbased fixation. Overall average motion error was low for both groups: less than $1 \mathrm{~mm}$ in the translational direction and less than or equal to $1^{\circ}$ in the rotational direction. There was a significant difference in the z-rotational direction between the two fixation groups. This error was small, approximately $1^{\circ}$, which is unlikely to be clinically significant. The resulting difference is likely due to statistical error or sampling basis. However, mask fixation demonstrated a significantly higher degree of variability in error, with a larger maximal displacement in each direction, compared to that with frame fixation.

While small, the setup error can occur for frame patients. In particular, frame slippage can occur in the time between frame placement and the start of treatment, leading to mistargeting of the lesion. ${ }^{14} \mathrm{Li}$ et al. measured the setup error for frame fixation using a modified GK Perfexion unit equipped with CBCT. ${ }^{9}$ They found the setup error to be low, less than $1 \mathrm{~mm}$ in the translational direction and less than $1^{\circ}$ in the rotational direction. Our results show similarly low setup errors using the GK Icon. For patients undergoing frame-based fixation on the GK Icon system, pretreatment CBCT scans can be obtained to confirm setup accuracy. Our results can be used as quality assurance thresholds. Several studies have measured setup motion uncertainties associated with the thermoplastic mask fixation used in LINAC-based systems. ${ }^{2,5,8,10,13,19}$ Kataria et al. found pretreatment uncertainties for frameless SRS to be (translational) $\mathrm{x}=-0.40 \pm 0.9 \mathrm{~mm}, \mathrm{y}=1.10 \pm 1.1 \mathrm{~mm}, \mathrm{z}$ $=-0.5 \pm 1.3 \mathrm{~mm}$, (rotational) $\mathrm{x}=-0.11 \pm 0.78^{\circ}, \mathrm{y}=0.2$ $\pm 0.44^{\circ}, \mathrm{z}=-0.29 \pm 0.35^{\circ} .5$ These results are within the range of the measured uncertainties in our study.

We also compared intrafraction motion uncertainties between frame-based and mask-based fixation. The intrafraction error was greater for mask patients than for frame patients in all three directions for both translation and rotation. Additionally, mask fixation demonstrated significantly higher variability than frame fixation. The 

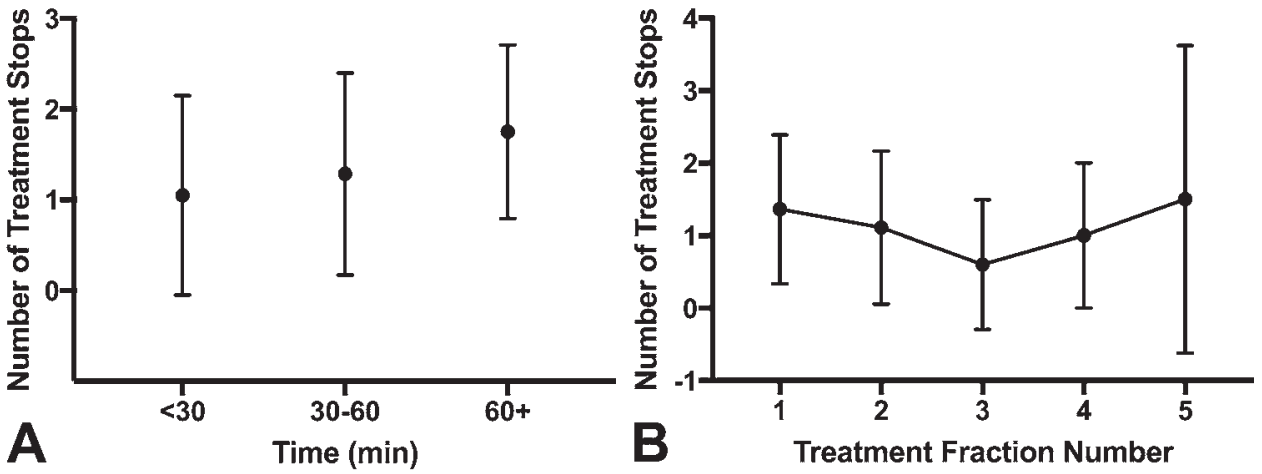

FIG. 3. A: Number of IR-based treatment stoppages based on treatment time. The number of treatment stoppages due to reaching the IR displacement threshold correlated with increasing treatment time. B: Number of IR-based treatment stoppages based on the treatment fraction number.

greater overall error and variance in the mask fixation group is likely due to several factors including conformity of the mask to the patient's face, amount of pressure applied by the mask on the skin, and deformations in mask assembly. ${ }^{10}$ Moreover, the ability of the patient to tolerate the procedure and limit their head movement can also affect accuracy. While CBCT allows for measuring motion error at the start and completion of treatment, it does not allow for real-time monitoring of motion during the treatment itself. Infrared tracking of a patient nose marker allows for real-time monitoring of head movement during treatment. Thermoplastic mask fixation in combination with IR tracking has been demonstrated to be sensitive for detecting motion at the nose tip. ${ }^{8}$ At our institution, we use an IR displacement threshold of $1.5 \mathrm{~mm}$. Once patient movement reaches this threshold, the treatment will be automatically stopped and CBCT can be performed to reset the patient into the desired position. A tighter threshold can be used to ensure less intrafractionation motion; however, this may increase the number of stoppages and thus prolong treatment time. We also measured the number of stoppages due to reaching the IR displacement threshold compared to the treatment fraction number. Over the course of the first 3 fractions, the number of treatment stoppages trended downward. We then saw an upward trend in stoppages for fractions 4 and 5 . Yet, these results can only be reported as observations given our limited number of cases. A larger number of patients will be needed to draw statistical conclusions from the data.

Furthermore, we stratified patients into two groups, metastases versus nonmetastases, to determine if the type of pathology being treated influenced the motion error. Overall, treatment pathology did not influence motion error. As in the primary analysis of all patients, mask fixation demonstrated translational and rotational setup errors similar to those for frame fixation but a higher degree of variability in error measurements for both metastases and nonmetastases groups. Also, intrafraction error and variance in error were significantly greater for mask fixation than for frame fixation for both groups. Comparing metastases and nonmetastases for frame or mask fixation alone did not show any differences in motion uncertainties.

As hypofractioned GK SRS becomes more prevalent, the use of noninvasive fixation will increase. While previ- ous studies using LINAC-based SRS have shown motion uncertainties to be equivalent between frame and mask fixation, GK SRS tends to involve longer treatment times and thus has a greater potential for motion error..$^{5,9}$ If motion error leads to decreased target accuracy, there is the potential for geographic mistargeting and potential injury to surrounding tissue. A better understanding of motion error associated with noninvasive fixation is essential. This is the first study to compare motion error associated with frameand mask-based fixation using the GK Icon. We found motion error to be low in both frame and mask fixation. However, mask fixation tended to demonstrate higher variability in the maximal displacement of error. This increased motion variability should be taken into account when treating small lesions or lesions near critical structures.

The present study has a few limitations. As a retrospective study, patients were not randomized to either frame- or mask-based fixation, resulting in potential selection bias. Also, this study focuses on setup and intrafraction uncertainties; however, there are other potential sources of error that contribute to the accuracy of GK treatment, including MRI-CT registration uncertainty, CBCT-CT registration uncertainty, and interobserver variation in target volume delineation. ${ }^{18}$ Chung et al. measured image coregistration error for frameless SRS using the GK Icon. ${ }^{1}$ They found that the uncertainties associated with MRI-CT registration and CBCT-CT registration were less than $1 \mathrm{~mm}$. We, therefore, made the assumption in our study that registration error has a minimal clinical impact. Additionally, the variability in error seen in mask fixation is directly dependent on the IR displacement threshold. If we were to lower the threshold to less than $1.5 \mathrm{~mm}$ of displacement, it would likely decrease error variability; however, treatment stoppages would increase and overall treatment time would be prolonged. The reverse would be seen if the threshold were to be increased. Therefore, we believe that using a displacement threshold of $1.5 \mathrm{~mm}$ results in submillimeter accuracy while limiting the number of treatment stoppages.

\section{Conclusions}

In summary, submillimeter motion uncertainty exists for frame-based and mask-based fixation using the GK Icon. Overall, mask fixation demonstrates a significantly 
higher degree of error variability than that with frame fixation both at setup and at intrafractionation. The variability in motion error associated with mask fixation must be taken into account when planning for small lesions or lesions near critical structures. Additionally, CBCT can be used to assess motion error for frame- and mask-based fixation and serve as a quality assurance tool.

\section{References}

1. Chung HT, Kim JH, Kim JW, Paek SH, Kim DG, Chun KJ, et al: Assessment of image co-registration accuracy for frameless gamma knife surgery. PLoS One 13:e0193809, 2018

2. Fuss M, Salter BJ, Cheek D, Sadeghi A, Hevezi JM, Herman TS: Repositioning accuracy of a commercially available thermoplastic mask system. Radiother Oncol 71:339-345, 2004

3. Gevaert T, Verellen D, Tournel K, Linthout N, Bral S, Engels B, et al: Setup accuracy of the Novalis ExacTrac 6DOF system for frameless radiosurgery. Int J Radiat Oncol Biol Phys 82:1627-1635, 2012

4. Gilbeau L, Octave-Prignot M, Loncol T, Renard L, Scalliet P, Grégoire V: Comparison of setup accuracy of three different thermoplastic masks for the treatment of brain and head and neck tumors. Radiother Oncol 58:155-162, 2001

5. Kataria T, Gupta D, Karrthick KP, Bisht SS, Goyal S, Abhishek A, et al: Frame-based radiosurgery: Is it relevant in the era of IGRT? Neurol India 61:277-281, 2013

6. Lamba M, Breneman JC, Warnick RE: Evaluation of imageguided positioning for frameless intracranial radiosurgery. Int J Radiat Oncol Biol Phys 74:913-919, 2009

7. Leksell L: The stereotaxic method and radiosurgery of the brain. Acta Chir Scand 102:316-319, 1951

8. Li W, Bootsma G, Von Schultz O, Carlsson P, Laperriere N, Millar BA, et al: Preliminary evaluation of a novel thermoplastic mask system with intra-fraction motion monitoring for future use with image-guided Gamma Knife. Cureus 8:e531, 2016

9. Li W, Cho YB, Ansell S, Laperriere N, Ménard C, Millar BA, et al: The use of cone beam computed tomography for image guided Gamma Knife stereotactic radiosurgery: initial clinical evaluation. Int J Radiat Oncol Biol Phys 96:214-220, 2016

10. Lightstone AW, Tsao M, Baran PS, Chan G, Pang G, Ma L, et al: Cone beam CT (CBCT) evaluation of inter- and intrafraction motion for patients undergoing brain radiotherapy immobilized using a commercial thermoplastic mask on a robotic couch. Technol Cancer Res Treat 11:203-209, 2012

11. Ma L, Chuang C, Descovich M, Petti P, Smith V, Verhey L: Whole-procedure clinical accuracy of gamma knife treatments of large lesions. Med Phys 35:5110-5114, 2008

12. Maciunas RJ, Galloway RL Jr, Latimer JW: The application accuracy of stereotactic frames. Neurosurgery 35:682-695, 1994

13. Minniti G, Clarke E, Lanzetta G, Osti MF, Trasimeni G, Bozzao A, et al: Stereotactic radiosurgery for brain metastases: analysis of outcome and risk of brain radionecrosis. Radiat Oncol 6:48, 2011
14. Otto K, Fallone BG: Frame slippage verification in stereotactic radiosurgery. Int J Radiat Oncol Biol Phys 41:199-205, 1998

15. Ruschin M, Nayebi N, Carlsson P, Brown K, Tamerou M, Li $\mathrm{W}$, et al: Performance of a novel repositioning head frame for Gamma Knife Perfexion and image-guided linac-based intracranial stereotactic radiotherapy. Int J Radiat Oncol Biol Phys 78:306-313, 2010

16. Salter BJ, Fuss M, Vollmer DG, Sadeghi A, Bogaev CA, Cheek DA, et al: The TALON removable head frame system for stereotactic radiosurgery/radiotherapy: measurement of the repositioning accuracy. Int J Radiat Oncol Biol Phys 51:555-562, 2001

17. Schlesinger D, Xu Z, Taylor F, Yen CP, Sheehan J: Interfraction and intrafraction performance of the Gamma Knife Extend system for patient positioning and immobilization. $\mathbf{J}$ Neurosurg 117 Suppl:217-224, 2012

18. Seravalli E, van Haaren PM, van der Toorn PP, Hurkmans CW: A comprehensive evaluation of treatment accuracy, including end-to-end tests and clinical data, applied to intracranial stereotactic radiotherapy. Radiother Oncol 116:131-138, 2015

19. Wong VY, Tung SY, Leung TW, Ho KH: CT verification of isocentre relocatability using stereotactic mask fixation system. Clin Oncol (R Coll Radiol) 15:280-287, 2003

20. Wu A: Physics and dosimetry of the gamma knife. Neurosurg Clin N Am 3:35-50, 1992

\section{Disclosures}

Dr. Danish receives an honorarium from Medtronic.

\section{Author Contributions}

Conception and design: Danish, Carminucci, Nie, Weiner. Acquisition of data: Carminucci, Nie. Analysis and interpretation of data: Carminucci, Nie, Weiner, Hargreaves. Drafting the article: Danish, Carminucci, Nie. Critically revising the article: Danish, Nie, Weiner, Hargreaves. Reviewed submitted version of manuscript: Danish, Carminucci, Nie, Weiner. Statistical analysis: Carminucci, Hargreaves. Study supervision: Danish.

\section{Supplemental Information}

\section{Online-Only Content}

Supplemental material is available with the online version of the article.

Supplemental Table and Figures. https://thejns.org/doi/ suppl/10.3171/2018.7.GKS181516.

\section{Previous Presentations}

This work was previously presented as an oral presentation at the 19th International Leksell Gamma Knife Society Meeting held in Dubai, United Arab Emirates, on March 4-8, 2018.

\section{Correspondence}

Shabbar F. Danish: Rutgers Cancer Institute of New Jersey, New Brunswick, NJ.danishsh@cinj.rutgers.edu. 